\title{
Genetic Polymorphisms of Xenobiotic Metabolizing Genes (GSTM1, GSTT1, GSTP1), Gene-Gene Interaction with Association to Lung Cancer Risk in North India; A Case Control Study
}

\author{
Ritambhara $^{1}$, Sonia Tiwari ${ }^{2}$, Sivakumar Vijayaraghavalu ${ }^{3}$, Munish Kumar ${ }^{1 *}$
}

\begin{abstract}
Aim: In this case control study involving, 220 human subjects; polymorphisms in xenobiotic metabolizing genes $(G S T-M 1,-T 1$ and $-P 1)$ and their association to lung cancer risk is being analysed among smokers and nonsmokers. GSTM1 or GSTT1 gene polymorphism and amino acid changes in GSTP1 have been correlated and may be associated to lung cancer risk. Other factor includes exposure to environmental pollutants and life style choices. We have explored gene-gene and gene-environment interaction in the aetiology of lung cancer risk among north Indian population. Patients and Methods: For the study we have collected 120 lung cancer patient blood samples from Kamala Nehru Memorial Cancer Hospital, Allahabad, Uttar Pradesh and 100 matched controls. DNA was isolated and GST-M1 and - T1 genotyping were assessed by multiplex PCR whereas the GSTP1 polymorphism was analysed using restriction fragment length polymorphism. The risk of lung carcinogenesis was assessed using logistic regression analysis calculating the odd ratio (OR) with $95 \%$ confidence interval (CI). Results: The risk of lung carcinogenesis was three fold higher for null GSTT1 (OR=3.045, 95\%CI=1.750-5.301, p-value $<0.001)$ genotype; whereas other two types; GSTM1 $(\mathrm{OR}=1.342,95 \% \mathrm{CI}=0.788-2.284$, p-value=0.270) and GSTP1 $(\mathrm{OR}=0.806,95 \% \mathrm{CI}=0.526-1.236$, $\mathrm{p}$-value $=0.323$ ) showed no association to lung cancer susceptibility respectively. Smokers diagnosed with lung cancer had more null genotypes for GSTT1 $(\mathrm{OR}=4.773,95 \% \mathrm{CI}=1.939-11.751, \mathrm{p}<0.001)$. The 'at risk' genotype combination GSTM1 (null) / GSTT1 (null) (OR=1.76, 95\%CI; 0.920-3.370, p-value=0.03) showed increased susceptibility to lung cancer risk. The genotype combination of GSTT1 (null)/GSTP1 (Ile/Ile) ( $\mathrm{p}=0.009)$ was associated with increased lung cancer risk. Conclusion: The results of this study suggest that; GSTT1 null genotype were more susceptible for lung cancer risk and smoking increases the susceptibility for lung cancer several folds among the North Indian population. Gene-gene interaction for null genotypes of GSTM1 and GSTT1 were correlated with higher risk of having lung cancer.
\end{abstract}

Keywords: GSTS- Polymorphism- GSTT1-GSTM1-GSTP1- Lung cancer

Asian Pac J Cancer Prev, 20 (9), 2707-2714

\section{Introduction}

Lung cancer is widespread malignancy in both men/women and reported increasing incidence rate and mortality among all cancer types across the world including India in last few decades. It is ranked fourth for new cases incidence and ranked third in number of death due to cancers (GLOBOCAN, WHO, 2018). Incidence of lung cancer in both sexes in Asia is $58.5 \%$, mortality $60.7 \%$ and 5 year prevalence is $56.6 \%$ which is higher than other cancers worldwide. New cases of lung cancer in worldwide outlines in 2018 in both sexes and all ages are $11.6 \%(2,093,876)$ in total reported $18,078,957$ cases (GLOBOCAN, WHO, 2018). Death cases reported according to GLOBOCAN 2018 data for both sexes and all ages are up to $18.4 \%(1,761,007)$ which is significantly higher in terms of mortality rate among all cancers. Lung cancer basically comprises of two types; non-small cell lung cancer (NSCLC) and small cell lung cancer (SCLC) in which $80 \%$ of occurrence is reported for NSCLC. Tobacco smoking is one of the leading causes for lung cancer related risks, but only 5-10\% smokers develop lung cancer in their life time. The various carcinogens present in tobacco and cigarette smoke are polycyclic aromatic hydrocarbons, benzo $[\alpha]$ pyrene, ethylene oxide, aldehydes and nitrosamine. These aromatic carcinogens are detoxified by cytochrome P450 gene and GSTs gene super family (Clement-Duchene et al., 2010, Garte et al., 2007).

${ }^{1}$ Department of Biochemistry, University of Allahabad, ${ }^{2}$ Department of Radiation Oncology, Kamala Nehru Memorial Hospital, Allahabad, UP-211002, ${ }^{3}$ Central Research Facility, Sri Ramachandra Institute of Higher Education and Research (Deemed to be University), Porur, Chennai, 600116, Tamil Nadu, India.*For Correspondence: munishkp@gmail.com 
Detoxification of carcinogens occurs in sequential phases includes phase I and phase II detoxification enzymes. Phase I detoxification enzymes makes catalytically active pro-carcinogens mediated through cytochrome P450 super gene family (Kiyohara et al., 2012). The GSTs (glutathione S-transferase) - GSTM1, GSTT1, and GSTP1 plays prominent role in metabolic detoxification, they are classified as phase II detoxification enzymes (Malik et al., 2010). They execute their role through conjugation to electrophilic metabolite with reduced glutathione. The carcinogens when not biotransformed gains ability to become activated consequently, forms DNA adduct which hampers genomic integrity leading to carcinogenesis. GSTM1 and GSTT1 are both expressed in lung tissues and play a crucial role in detoxification of cigarette smoke in lungs. The impaired functioning of null genotypes of GSTM1 and GSTT1 is unable to prevent formation of DNA adducts and subsequently leading to promotion of carcinogenesis. DNA adducts level found to be significantly increased with association to gene polymorphism of GSTT1, GSTM1 and GSTP1 (Lee et al., 2010). GSTP1 has nucleotide transition from A to $\mathrm{G}$ in exon 5 (codon 105) occur changes in isoleucine (Ile) to valine (Val) affects the enzymatic activity and increased susceptibility towards cancer (Mota et al., 2015, Vettriselvi et al., 2006). GSTP1 functions in detoxification of compounds known to cause oxidative stress like thymidine and uracil propanol (Berhane et al., 1994). Studies on GSTs gene polymorphisms among North Indian population are limited and inconsistent findings obtained across Indian region. So there is need to explore gene-gene and gene-environment interaction to find its association to lung cancer risk. We hypothesized in present case control study that deletion polymorphism of GSTT1 and GSTM1 higher in lung cancer patients compared to controls as well as with relation to smoking. We have also explored GSTP1 genotypes distribution in cases and controls whether there is more susceptibility towards lung cancer risk of other alleles and genotypes.

\section{Materials and Methods}

\section{Study Subjects}

In this study both the cancer patients $(\mathrm{n}=120)$ and matched controls (without cancer; $\mathrm{n}=100$ ) were recruited from Kamala Nehru Memorial Cancer Hospital, Uttar Pradesh, India. Based on the bronchoscopy and histological studies from the lung biopsy; the cancer patients were divided into following; adenocarcinoma $(\mathrm{n}=76)$, squamous cell carcinoma $(\mathrm{n}=28)$ and small cell lung carcinoma $(\mathrm{n}=16)$ according to medical records of patients. The ethnic North Indians who were unrelated to cancer patients served as controls. The study was conducted (February 2017 to February 2019) post approval from Population Resource and Research centre, Allahabad Institute Ethical Committee (IERB Reference: 18/9.39) and with prior consent from the participants. Also, ethical principles as stated in Declaration of Helsinki were strictly followed while handling human subjects. Questionnaires for the control and cancer patients were prepared both in local language and English. It includes ethnicity, smoking habits, dietary habits, socio-economic status, occupation and familial history. The lung cancer patients were recruited without any prior neoadjuvant therapy. The sample size taken for this study was appropriate to strengthen $80 \%$ statistical power for allele frequencies ranges between $11-35 \%$ to estimate odd ratios greater than 1.34 or lower than 0.69 for finding risk associated to lung cancer.

Inclusion and exclusion criteria for lung cancer patients and controls are as follows:

\section{Inclusion criteria}

- Ethnic North Indians in the age range of 18 to 75 with lung cancers.

- Patients smoking $\geq 10$ cigarettes/day for at least 20 years.

- Non-smoking cancer patients those who never smoked less than a one pack year or 20 cigarettes per day.

- Patients undergone bronchoscopy.

- Age, sex and ethnicity matched healthy individuals without reported any kinds of cancer (s) were included as controls.

\section{Exclusion criteria}

Men and women greater than 75 years and/or with double metastasis and/or diagnosed with any other diseases or disorders were excluded from the study. As well both the cancer patients and controls that were not willing to participate in the study were excluded.

\section{DNA extraction and genotyping}

$2 \mathrm{ml}$ of peripheral blood samples withdrawn for genomic DNA isolation, using Qaigen (Germany) DNA blood mini isolation kit following the manufacturers' protocol and quantified on nano-drop spectrophotometer (Agilent technologies, Santa Clara, USA) from both cancer patients and control subjects. The DNA samples were stored at $-80^{\circ} \mathrm{C}$ until further use.

\section{GSTM1 and GSTT1 genotyping}

Multiplex PCR was performed to analyse null genotypes of GSTM1 and GSTT1 gene polymorphism as described earlier (Rehman et al., 1996). Briefly, to each well in a PCR plate, $25 \mu 1$ (2X PCR master mixes, Sigma Aldrich, St. Louis, USA), 50-100 ng of DNA and 10 pmol of primers were added and the reaction was set with its respective positive and negative controls in a thermal cycler 8800 (Agilent Technologies, Australia). PCR cycling conditions: initial denaturation at $94{ }^{\circ} \mathrm{C}$ for 5 min, followed by denaturation $94^{\circ} \mathrm{C}$ for $1 \mathrm{~min}$, annealing $59^{\circ} \mathrm{C}$ for $1 \mathrm{~min}$ and extension $72^{\circ} \mathrm{C}$ for $10 \mathrm{~min}$. The PCR products were then electrophoresed using $2 \%$ agarose gel stained with ethidium bromide and visualized using Geldoc XR+ (Biorad System, Canada) system. Bands at 215- and $480 \mathrm{bp}$ were considered as positive for GSTM1 and GSTT1 genotypes respectively, while CYPA1 yielded band of $315 \mathrm{bp}$ (Figure 1). 
Primer sequences used were as follows

GSTT1 (Forward), 5'TTCCTTACTGGTCCTCACATCTC 3' (Reverse), 5'TCACCGGATCATGGCCAGCA 3'

GSTM1 (Forward), 5'GAACTCCCTGAAAAGCTAAAGC 3'

(Reverse), 5 'GTTGGGCTCAAATATACGGTGG 3'

\section{GSTP1 genotyping}

GSTP1genotyping performed by PCR-RFLP method as described earlier (Harries et al., 1997) using following primer sequences:

Forward, 5'-ACCCCAGGGCTCTATGGGAA-3';

Reverse, 5'-TGAGGGCACAAGAAGCCCCT-3'

The PCR conditions used were as follows: Initial denaturation at $95^{\circ} \mathrm{C}$ for $5 \mathrm{~min}$, followed with denaturation of $94^{\circ} \mathrm{C}$ for $30 \mathrm{sec}$, annealing $58^{\circ} \mathrm{C}$ for 45 sec for 35 cycles and final extension at $72{ }^{\circ} \mathrm{C}$ for $10 \mathrm{~min}$. The amplified product (176bp) was digested by incubating overnight with BsmA1 (New England Biolabs, USA) at $37{ }^{\circ} \mathrm{C}$ and electrophoresed using $10 \%$ polyacrylamide gel. The amplified PCR product of $176 \mathrm{bp}$ obtained for GSTP1 by $2 \%$ gel electrophoresis (Figure 2A). After performing $10 \%$ native PAGE ; the band pattern showed $176 \mathrm{bp}$ undigested product obtained for Ile/Ile (wild type) genotype, 91 and 85 bp genotype showed mutant genotype Val/Val and three fragment obtained for Ile/Val genotype 176, 91 and 85 bp respectively(Figure $2 \mathrm{~B}$ ). Then the ethidium bromide staining was done and visualized using Geldoc system (Bio Rad system, Canada).

\section{Statistical analysis}

The gene polymorphisms of GSTT1, GSTM1, GSTP1 distribution in North Indian population assessed by unconditional logistic regression used for evaluation of odd ratio (OR) and $95 \%$ confidence interval (CI) used to describe the strength of association with disease. Binary Logistic Regression model used to evaluate the difference in prevalence and association between cases and groups. p-value $<0.05$ was considered as statistically significant. Statistical analysis was performed using SPSS software 16.00 (SPSS In; Chicago, IL, USA).

\section{Results}

In present study we assessed the role of xenobiotic

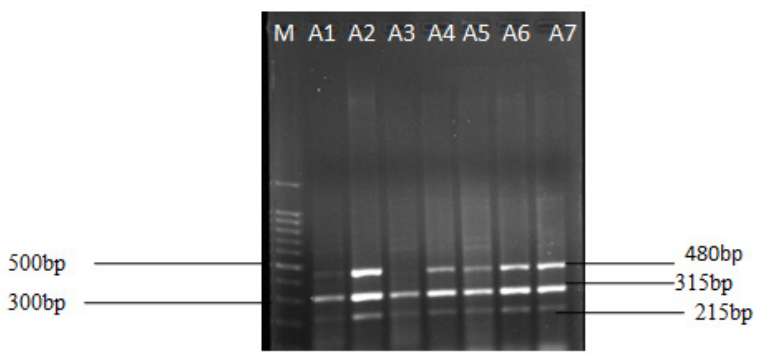

Figure 1. Multiplex PCR for GSTT1 and GSTM1. 2\% gel electrophoresis of GSTM1 and GSTT1 genotype. Lane $\mathrm{M}$, base pair marker (ProxiO, DNA marker). Lane A1 and A3 represents deletion of GSTT1 (480bp), Lane A1 to A7 showed presence of GSTM1 (215bp), CYP1A1 (315) serves as internal control.

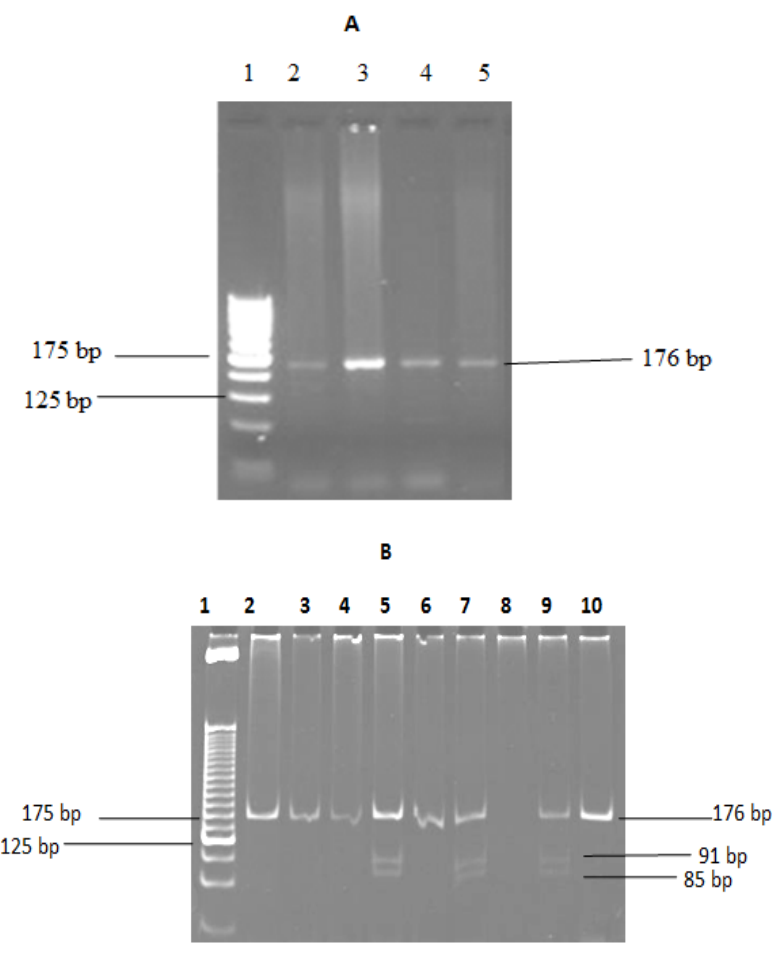

Figure 2. Genetic Polymorphism Using Restriction Fragment Length Polymorphism, Native 10\% PAGE, RFLP Products of GSTP1. A, Lane 1 showed 25bp gene marker, lane 2, 3, 4 and 5 showed 176 bp PCR products ( $2 \%$ agarose gel electrophoresis); B, Lane 1 showed $25 \mathrm{bp}$ gene marker, Lane 2, 3, 4,6 and 10 (Ile/Ile) genotype and Lane 5, 7, 9 (Ile/Val) genotype RFLP product (Native $10 \%$ PAGE).

metabolizing genes, age, gender, smoking and histology with relation to lung cancer risk. The age of lung cancer cases (mean \pm SD, 58.41 \pm 10.49 , Table 1) including 104 males and 16 females found comparatively higher than controls $(51.51 \pm 10.76)$ and statistically significant in both gender (Table 1). The histology data obtained from medical records of lung cancer patients having squamous cell carcinoma (SCC, 23.34\%), adenocarcinoma (ADCC, $63.34 \%$ ) and small cell carcinoma (SCLC, 13.34\%) (Table 1). It was observed in present study that most of lung cancer patients have TNM staging I (56.67\%) and II $(28.34 \%)$ (Table 1$)$. Out of 120 lung cancer patients $93.3 \%$ were smokers and $6.67 \%$ non-smokers which was significantly higher than that of controls (Table 1). The genotype frequencies in lung cancer patients obtained for GSTT1 (positive, 40\%), GSTT1 (null, 60\%) and in controls GSTT1 (positive, 67\%), GSTT1 (null, $33 \%$ ) (Table 2). GSTT1 null (60\%) genotype of lung cancer cases were higher comparatively to controls (33\%, Table 2). We found increased GSTM1 deletion polymorphism in lung cancer cases $(53.34 \%)$ than controls (46\%, Table 2). The odd ratios found for GSTT1 $(\mathrm{OR}=3.04, \mathrm{CI}=1.750-5.301, \mathrm{p}<0.001$, Table 2) showed strong association whereas GSTM1 $(\mathrm{OR}=1.342, \mathrm{CI}=0.788$ $2.284, \mathrm{p}=0.270$, Table 2 ) showed no association. The null genotype for both GSTM1 and GSTT1 with estimated odd ratio $(\mathrm{OR}=1.76,95 \% \mathrm{CI}$ : $1.030-3.012, \mathrm{p}=0.03$, Table 5) found to be statistically significant in lung cancer patients. The odd ratio estimated for smokers in lung cancer cases 
Table 1. Demographic Variables of Lung Cancer Cases and Controls, * All p-values $<0.05$ was Considered Statistically Significant

\begin{tabular}{|c|c|c|c|c|}
\hline Demographic Variables & Cases, $\%(\mathrm{~N}=120)$ & Controls, $\%(\mathrm{~N}=100)$ & Chi square $(x 2)$ & P-value \\
\hline Age (mean \pm SD) & $58.19 \pm 10.49$ & $51.51 \pm 10.76$ & & \\
\hline \multicolumn{5}{|l|}{ Gender } \\
\hline Male & $104(86.67)$ & $72(72.0)$ & 1.240 & $<0.001^{*}$ \\
\hline Female & $16(13.34)$ & $28(28.0)$ & 36.00 & $<0.001^{*}$ \\
\hline \multicolumn{5}{|l|}{ Smoking status } \\
\hline Smokers & $112(93.3)$ & $26(26.0)$ & 82.00 & $<0.001^{*}$ \\
\hline Non-smokers & $8(6.67)$ & $74(74.0)$ & 78.00 & $<0.001^{*}$ \\
\hline \multicolumn{5}{|l|}{ Histology types } \\
\hline SQCC & $28(23.34)$ & & & \\
\hline $\mathrm{ADCC}$ & $76(63.34)$ & & & \\
\hline SCLC & $16(13.34)$ & & & \\
\hline \multicolumn{5}{|l|}{ TNM staging } \\
\hline I & $8(6.67)$ & & & \\
\hline II & $34(28.34)$ & & & \\
\hline III & $10(8.34)$ & & & \\
\hline IV & $68(56.67)$ & & & \\
\hline \multicolumn{5}{|l|}{ Lymph node involvement } \\
\hline No & $46(38.34)$ & & & \\
\hline N1 & $16(13.34)$ & & & \\
\hline $\mathrm{N} 2$ & $18(15)$ & & & \\
\hline N3 & $26(21.67)$ & & & \\
\hline $\mathrm{N} 4$ & $14(11.67)$ & & & \\
\hline \multicolumn{5}{|l|}{ Metastasis } \\
\hline M0 & $24(20)$ & & & \\
\hline M1 & $76(63.34)$ & & & \\
\hline Unknown & $20(16.67)$ & & & \\
\hline
\end{tabular}

with association to GSTT1 null genotypes (OR=4.773, $95 \% \mathrm{CI}=1.939-11.751, \mathrm{p}<0.001)$ found statistically significant whereas no association found with GSTM1 $(\mathrm{OR}=2.00,95 \% \mathrm{CI}=0.166-1.26$, $\mathrm{p}$-value $=0.123$, Table 4$)$. In smokers having lung cancer both GSTT1 (80.35\%) and GSTM1 (73.21\%) null genotypes were higher than controls of GSTT1 (46.15\%) and GSTM1 (57.69\%) (Table 4). The genotype frequency of GSTP1 $(\mathrm{OR}=0.806,95 \% \mathrm{CI}=0.526-1.236 \mathrm{p}=0.323$, Table 3$)$ showed no association to lung cancer risk. In cases genotype frequency for GSTP1; AA (60\%), AG (33.34\%), GG $(6.67 \%)$ in which AG (Ile/Val, 41\%) was lower in lung cancer cases comparatively to controls (Table 3 ). Allele frequencies of lung cases in GSTP1 showed that allele A $(76.67 \%)$ higher than controls $(72.5 \%)$ and allele
G (23.34\%) lower than controls (27.5\%) (Table 3). The genotype AG (Ile/Val) of GSTP1 found to be lower in smokers of lung cancer cases $(33.03 \%$, Table 4$)$ than controls $(57.69 \%$, Table 4). GSTP1 genotype showed no association to smoking $(\mathrm{OR}=0.434,95 \%$ $\mathrm{CI}=0.206-0.916 \mathrm{p}$-value $=0.02$, Table 4$)$. The genotype combination of GSTT1 (null) and GSTP1 (Ile/Ile) showed significant association $(\mathrm{OR}=2.030,95 \% \mathrm{CI}=1.196-3.448$, $\mathrm{p}$-value $=0.009$, Table 5). Genotype combinations of GSTM1 (positive) / GSTT1 (null) $(\mathrm{OR}=1.5,95 \% \mathrm{CI}=0.887$ 2.5360 ) and GSTM1 (null)/GSTT1 (positive) $(\mathrm{OR}=1.174$, $95 \% \mathrm{CI}=0.670-2.057$ ) (Table 5) were associated with lung cancer risk but statistically non significant.

Genotypic combination of GSTP1(Ile/Val+Val/Val) / GSTM1(null) (OR=1.45, 95\%=0.840-2.484), GSTP1(Ile/

Table 2. Genotype Frequency with Odd Ratio of GSTT1 and GSTM1, *All p-values $<0.05$ was Considered Statistically Significant

\begin{tabular}{|c|c|c|c|c|}
\hline Genotype Frequency & GSTT1 (+) & GSTT1 (-) & $\mathrm{P}$-value & OR $(95 \% \mathrm{CI})$ \\
\hline Controls (\%) & $67(67)$ & $33(33)$ & 1.0 & 1.0 \\
\hline \multirow[t]{2}{*}{ Cases $(\%)$} & $48(40)$ & $72(60)$ & $<0.001$ & $3.045(1.750-5.301)$ \\
\hline & GSTM1 $(+)$ & GSTM1 (-) & & \\
\hline Controls (\%) & $54(54)$ & $46(46)$ & 1.0 & 1.0 \\
\hline Cases (\%) & $56(46.67)$ & $64(53.34)$ & 0.270 & $1.342(0.788-2.284)$ \\
\hline
\end{tabular}


Table 3. Genotype and Allele Frequency with Odd Ratio of GSTP1

\begin{tabular}{|c|c|c|c|c|c|}
\hline GSTP1 & AA & $\mathrm{AG}$ & GG & P-value & OR $(95 \% \mathrm{CI})$ \\
\hline \multicolumn{6}{|l|}{ Genotype Frequency } \\
\hline Controls $n=100(\%)$ & $52(52.0)$ & $41(41.0)$ & $7(7.0)$ & 1.0 & 1.0 \\
\hline Cases $=120(\%)$ & $72(60)$ & $40(33.34)$ & $8(6.67)$ & 0.323 & $0.806(0.526-1.236)$ \\
\hline Chi square $\left(x^{2}\right)=1.499$ & \multicolumn{5}{|c|}{$P-$ value $=0.473$} \\
\hline Allele Frequency & A & G & p-value & & OR $(95 \% \mathrm{CI})$ \\
\hline Controls $(\%)$ & $145(72.5)$ & $55(27.5)$ & 1.0 & & 1.0 \\
\hline Cases $(\%)$ & $184(76.67)$ & $56(23.34)$ & 0.517 & & $5(0.559-1.340)$ \\
\hline
\end{tabular}

Table 4. Correlation of Smokers and Non-Smokers Status to GSTM1, GSTT1 and GSTP1 Genotype, *All p-values $<0.05$ was Considered Statistically Significant

\begin{tabular}{|c|c|c|c|c|}
\hline \multirow[t]{2}{*}{ Gene } & \multicolumn{4}{|l|}{ Smokers } \\
\hline & Cases $(\%)$ & Controls $(\%)$ & OR $(95 \% \mathrm{CI})$ & $\mathrm{P}$-value \\
\hline \multicolumn{5}{|l|}{ GSTM1 } \\
\hline Present & $30(26.78)$ & $11(42.30)$ & 1.0 & 1.0 \\
\hline Absent & $82(73.21)$ & 15 (57.69) & $2.00(0.829-4.848)$ & 0.123 \\
\hline \multicolumn{5}{|l|}{ GSTT1 } \\
\hline Present & $22(19.64)$ & $14(53.84)$ & 1.0 & 1.0 \\
\hline Absent & & $12(46.15)$ & $4.773(1.939-11.751)$ & $<0.001$ \\
\hline GSTP1 & $90(80.35)$ & & & \\
\hline AA & $72(64.28)$ & $10(38.46)$ & 1.0 & 1.0 \\
\hline $\mathrm{AG}$ & $37(33.03)$ & $15(57.69)$ & $0.434(0.206-0.916)$ & 0.029 \\
\hline \multirow[t]{2}{*}{ GG } & $3(2.67)$ & $1(3.84)$ & & \\
\hline & Non-Smokers & & & \\
\hline GSTM1 & Cases $(\%)$ & Controls $(\%)$ & OR $(95 \% \mathrm{CI})$ & P-value \\
\hline Present & $4(50)$ & 54 (72.97) & 1.0 & 1.0 \\
\hline Absent & $4(50)$ & $20(27.02)$ & $2.7(0.616-11.835)$ & 0.188 \\
\hline \multicolumn{5}{|l|}{ GSTT1 } \\
\hline Present & $6(75)$ & $59(79.72)$ & 1.0 & 1.0 \\
\hline Absent & $2(25)$ & $15(20.27)$ & $1.311(0.240-7.160)$ & 0.754 \\
\hline \multicolumn{5}{|l|}{ GSTP1 } \\
\hline $\mathrm{AA}$ & $4(50)$ & $44(59.45)$ & 1.0 & 1.0 \\
\hline $\mathrm{AG}$ & $3(37.5)$ & $28(37.83)$ & $1.739(0.523-5.785)$ & 0.367 \\
\hline GG & $1(12.5)$ & $2(2.70)$ & & \\
\hline
\end{tabular}

Ile) $/ G S T M 1($ null $)(\mathrm{OR}=1.34,95 \% \mathrm{CI}=0.780-2.280)$, GSTP 1 (Ile/Ile+Val/Val)/GSTM1 (positive) $(\mathrm{OR}=1.167,95 \% \mathrm{CI}=0.677-2.011), \mathrm{GSTP} 1(\mathrm{Ile} / \mathrm{Ile}) /$ GSTT1 (null) $(\mathrm{OR}=1.5,95 \% \mathrm{CI}=0.883-2.549)$ and GSTP1 (Ile/Val+Val/Val)/GSTT1(null) (OR=1.62,95\% $\mathrm{CI}=0.951-2.76)$ (Table 5) showed association but statistically non significant. GSTM1 (null)/GSTP1 (Ile/Ile) $(\mathrm{OR}=1.174,95 \% \mathrm{CI}=0.704-1.957)$, GSTT1 (null)/GSTP1 $(\mathrm{Ile} / \mathrm{Val}+\mathrm{Val} / \mathrm{Val})(\mathrm{OR}=1.354,95 \% \mathrm{CI}=0.778-2.356)$ (Table 5) correlated to lung cancer risk and statistically non- significant.

\section{Discussion}

GSTs are phase II metabolizing enzymes, it consist of several forms of GSTs such as GSTA (alpha), GSTT1 (theta), GSTM1 (mu) and GSTP1 (pi), and have role in biotransformation of carcinogens. They are the candidate genes to detoxify carcinogenic compounds and thus show susceptibility towards lung cancer risk because of their impaired ability (Ihsan et al., 2014). The function of all GSTs is to metabolize the carcinogens via conjugating to glutathione and then converting into hydrophilic metabolites and eliminating it from the body. The genotype frequency of these GSTs varies in Indian subcontinents. In present study, we have assessed the role of GSTs polymorphism with association to lung cancer risk with three polymorphic genotypes of GSTT1, GSTM1, and GSTP1. Our findings suggested that the GSTT1 holds positive association towards lung cancer risk whereas GSTM1 and GSTP1 showed no association to lung cancer risk in this study. The genotype combination of GSTM1 (null)/GSTT1 (null) and GSTT1 (null)/GSTP1 (Ile/Ile) showed increased susceptibility towards lung cancer. In present study the age mean differences between lung cancer cases and control were higher suggesting that it is 
Table 5. Genotype Combinations of GSTM1, GSTT1 and GSTP1, *All p-values $<0.05$ was Considered Statistically Significant

\begin{tabular}{|c|c|c|c|c|c|}
\hline Genotype Combination & & Control (n \%) & Cases (n \%) & OR (95\% Confidence interval) & $\mathrm{P}$-value \\
\hline GSTM1 & GSTT1 & & & & \\
\hline Positive & Positive & $54(54)$ & $48(40)$ & 1.0 & 1.0 \\
\hline Null & Null & $46(46)$ & $72(60)$ & $1.761(1.030-3.012)$ & $0.039 *$ \\
\hline Positive & Null & $54(54)$ & $72(60)$ & $1.5(0.887-2.5360$ & 0.130 \\
\hline Null & Positive & $46(46)$ & $48(40)$ & $1.174(0.670-2.057)$ & 0.575 \\
\hline GSTM1 & GSTP1 & & & & \\
\hline Positive & Ile/Ile & $54(54)$ & $72(60)$ & 1.0 & 1.0 \\
\hline Null & $\mathrm{Ile} / \mathrm{Val}+\mathrm{Val} / \mathrm{Val}$ & $46(46)$ & $48(40)$ & $0.783(0.458-1.339)$ & 0.371 \\
\hline Null & Ile/Ile & $46(46)$ & $72(60)$ & $1.174(0.704-1.957)$ & 0.539 \\
\hline Positive & Ile/Val+ Val/Val & $54(54)$ & $48(40)$ & $0.667(0.394-1.127)$ & 0.130 \\
\hline GSTT1 & GSTP1 & & & & \\
\hline Positive & Ile/Ile & $67(67)$ & $72(60)$ & 1.0 & 1.0 \\
\hline Null & $\mathrm{Ile} / \mathrm{Val}+\mathrm{Val} / \mathrm{Val}$ & $33(33)$ & $48(40)$ & $1.354(0.778-2.356$ & 0.284 \\
\hline Null & Ile/Ile & $33(33)$ & $72(60)$ & $2.030(1.196-3.448)$ & $0.009^{*}$ \\
\hline Positive & Ile/Val+ Val/Val & $67(67)$ & $48(40)$ & $0.667(0.405-1.097)$ & 0.111 \\
\hline GSTP1 & GSTM1 & & & & \\
\hline Ile/Ile & Positive & $52(52)$ & $48(40)$ & 1.0 & 1.0 \\
\hline $\mathrm{Ile} / \mathrm{Val}+\mathrm{Val} / \mathrm{Val}$ & Null & $48(48)$ & $64(53.34)$ & $1.444(0.840-2.484)$ & 0.184 \\
\hline Ile/Ile & Null & $52(52)$ & $64(53.34)$ & $1.333(0.780-2.280$ & 0.293 \\
\hline Ile/Ile+ Val/Val & Positive & $48(48)$ & $56(46.67)$ & $1.167(0.677-2.011)$ & 0.579 \\
\hline GSTP1 & GSTT1 & & & & \\
\hline Ile/Ile & Positive & $52(52)$ & 48 & 1.0 & 1.0 \\
\hline $\mathrm{Ile} / \mathrm{Val}+\mathrm{Val} / \mathrm{Val}$ & Null & $48(48)$ & $72(60)$ & $1.625(0.951-2.777)$ & 0.076 \\
\hline Ile/Ile & Null & $52(52)$ & $72(60)$ & $1.5(0.883-2.549)$ & 0.134 \\
\hline Ile/Ile+ Val/Val & Positive & $48(48)$ & $48(40)$ & $1.083(0.619-1.897)$ & 0.779 \\
\hline
\end{tabular}

an old stage disease in which DNA adducts accumulation occurs due to smoking and environmental pollutants exposure develops lung cancer risk as impaired functions of GSTs. In normal individuals of south Indian population frequency of deletion for GSTT1 is reported as (30.4\%) and GSTM1 (16.8\%) whereas in present north Indian population study null genotypes of GSTT1 and GSTM1 reported in control subjects 33\% and $46 \%$ respectively (Naveen et al., 2004). Bag et al., (2014) showed in North Indian population increased susceptibility of lung cancer patients having GSTT1 null genotypes in cases (23\%) than controls (15\%), similarly we also found increased frequency of GSTT1 null genotypes $60 \%$ in cases than $(33 \%)$ controls, and these results suggest that ethnicity and life style choice have prominent role in aetiology of lung cancer. We also found no association ( $\mathrm{p}$-value $=0.367$ ) with GSTP1 Ile/Val genotype in non- smokers similar to North Eastern region of India where it was found same with non-smokers (Ihsan et al., 2011).

Recent study in Iranian population showed strong correlation of GSTT1 $(\mathrm{OR}=2.4,95 \% \mathrm{CI}=1.32-4.35$, $\mathrm{p}$-value $=0.005)$ and no association to GSTM1 $(\mathrm{OR}=1.33$, $95 \% \mathrm{CI}=0.79-2.25$, $\mathrm{p}$-value $=0.35$ ) similar to our findings in north Indian population (Adibhesami et al., 2018). Our study showed positive association to lung cancer risk with genotype combination of GSTM1 (null) and GSTT1 (null)
(OR=1.76; 95\% CI=1.030-3.012), showed previously by Cajas-Salazar et al., 2003 (OR=2.32; CI=1.01-6.04). In Caucasian population Lewis et al., 2002 showed lower lung cancer risk with association to GSTM1 null genotype $(\mathrm{OR}=0.50 ; 95 \% \mathrm{CI}=0.29-0.87)$ similar to our results showed decreased association in North Indian population $(\mathrm{OR}=1.34 ; 95 \% \mathrm{CI}=0.788-2.284)$. Researchers from North Eastern region of India found no association to GSTT1 $(\mathrm{OR}=0.32,95 \% \mathrm{CI}=0.15-0.71$, $\mathrm{p}$-value $=0.13)$ contrary to our result we found strong association to GSTT1 $(\mathrm{OR}=3.045,95 \% \mathrm{CI}=1.750-5.301$, $\mathrm{p}$-value $<0.001)$. Both the regions of India have different geography, climate and life style differences pose strong effect of GSTs gene polymorphism in lung cancer risk (Ihsan et al., 2014).

Shukla et al., (2013) reported significant association in north Indian population GSTT1 null genotypes $(\mathrm{OR}=1.87$, 95\% CI: $1.25-2.80, \mathrm{p}=0.002)$ whereas GSTM1 null genotypes $(\mathrm{OR}=1.03, \mathrm{CI}: 0.71-1.51, \mathrm{p}=0.875)$ which was consistent to present study.

Our study finds strong correlation with genotype combination of GSTM1 (null)/GSTT1 (null) (p-value=0.03) and GSTT1 (null)/GSTP1 (Ile/Ile) (p-value=0.009) showed similarity to Chen et al., 2006 reported values GSTM1 (null)/GSTT1 (null) (p-value=0.000), GSTP1 (Ile/Val+Val/ Val) and GSTT1 (null) (p-value=0.003).

A study in north Indian population by Sharma et al., 
(2015) found that 'at risk' genotypes of null GSTM1 and GSTT1 are strongly associated to lung cancer risk which was in favour of our findings. The genotype combination of null GSTT1 and GSTP1 Ile/Ile genotypes $(\mathrm{p}=0.0001)$ have two times increased risk of having lung cancer similar to our study. They found GSTM1 null genotypes $(\mathrm{OR}=1.65,95 \% \mathrm{CI}=116-2.3)$ was associated to lung cancer risk whereas we found no association. Similar to our study Jiang et al., (2014) showed null genotype of GSTT1 $(\mathrm{OR}=1.574,95 \% \mathrm{CI}=1.044-2.372$, p-value=0.03) in smokers are positively correlated to lung cancer risk and in our study GSTT1 (OR=4.773 95\% $\mathrm{CI}=1.930-11.751$, $\mathrm{p}<0.001$ ) associated with four fold increased risk of having lung cancer in North Indian population.

In contradictory to our study Nasir Uddin et al., (2014) reported no association of GSTT1 $(\mathrm{OR}=0.84,95 \% \mathrm{CI}$ : $0.46-1.55, \mathrm{p}=0.573)$ and GSTM1 $(\mathrm{OR}=1.06,95 \% \mathrm{CI}$ : $0.62-1.81, \mathrm{p}=0.820$ ) as we find association with GSTT1 genotypes whereas they found strong association of GSTP1 (OR=3.56, 95\%CI: 1.70-7.46, p=0.0001) and we found no association. In similarity to our results, South Indian study confirmed two fold increased risk of having lung cancer with GSTT1 null genotypes (Peddireddy et al., 2016). The limitation of the present study is that it's small sample size and few genes chosen for study.

The main outcome of this study suggested that GSTT1 was the prominent candidate gene and has three-fold increased susceptibility to lung cancer risk in North Indian population. Different genotype combinations posing positive correlation to promote lung cancer risk. Genotype combination of GSTM1 (null)/GSTT1 (null) and GSTT1 (null)/GSTP1 (Ile/Ile) considered to be 'at risk' genotypes showed strong association towards risk of lung cancer in north Indian population. They can be taken into account for detection for lung cancer tumorigenesis and overall risk factor generation. It can serve as prognostic marker in lung carcinogenesis. There are various conflicting results obtained in various population of Indian population which showed that life style choices and environmental exposure are key players in posing lung cancer risk.

\section{Acknowledgements}

The authors would like to thanks Department of Science and Technology (DST) and UGC for funding to support this work. Ritambhara is thankful to UGC fellowship.

\section{Conflict of interest}

None declared.

\section{References}

Abdel-Rehman SZ, el-Zein RA, Anwar WA, Au WW (1996). Multiplex PCR procedure for polymorphic analysis of GSTM1 and GSTT1 gene in population studies. Cancer Lett, 107, 229-33.

Adibhesami G, Shahsavari GR, Amiri A, et al (2018). Glutathione S-transferase M1 (GSTM1) and T1 (GSTT1) polymorphisms and lung cancer risk among a select group of Iranian people. Asian Pac Cancer Prev, 19, 2921-7.
Bag A, Bag N, Jeena LM, Jyala NS (2014). Glutathione S-transferase T1 and myeloperoxidase $-463 \mathrm{G}>$ A genotypes in lung cancer patients of Kumaun region. J Nat Sci Biol Med, 5, 293-6.

Berhane K, Widersten M, Engstrom A, Kozarich JW, Mannervik B (1994). Detoxication of base propenals and other alpha, beta-unsaturated aldehyde products of radical reactions and lipid peroxidation by human glutathione transferases. Proc Natl Acad Sci U S A, 91, 1480-4.

Cajas-Salazar N, Sierra-Torres CH, Salama SA, Zwischenbergere JB, Au WW (2003). Combined effect of MPO, GSTM1 and GSTT1 polymorphism on chromosome aberrations and lung cancer risk. Int J Hyg Environ Health, 206, 473-83.

Carlsten C, Sagoo GS, Frodsham AJ, Burke W, Higgins JP (2008). Glutathione S-transferase M1 (GSTM1) polymorphisms and lung cancer: a literature-based systematic HuGE review and meta analysis. Am J Epidemiol, 167, 759-74.

Chen HC, Cao YF, Hu WX, et al (2006). Genetic polymorphisms of phase II metabolic enzymes and lung cancer susceptibility in a population of Central South China. Dis Markers, 22, 141-52.

Clément-Duchêne C, Vignaud JM, Stoufflet A, et al (2010). Characteristics of never smoker lung cancer including environmental and occupational risk factors. Lung Cancer, 67, 144-50.

Garte S, Taioli E, Popov T, et al (2007). Role of GSTT1 deletion in DNA oxidative damage by exposure to polycyclic aromatic hydrocarbons in humans. Int $J$ Cancer, 120, 2499-503.

GLOBOCAN (2018). World Health Organization.

Harries LW, Stubbins MJ, Forman D, Howard GC, Wolf CR (1997). Identification of genetic polymorphism at the glutathione S-transferase Pi locus and association with susceptibility to bladder, testicular and prostate cancer. Carcinogenesis, 18, 641-4.

Ihsan R, Chauhan PS, Mishra AK, et al (2011). Multiple analytical approaches reveal distinct gene- environment interactions in smokers and non smokers in lung cancer. PLoS One, 6, 29431.

Ihsan R, Chauhan PS, Mishra AK, et al (2014). Copy number polymorphism of glutathione-S-transferase genes (GSTM1 \& GSTT1) in susceptibility to lung cancer in a high-risk population from north-east India. Indian J Med Res, 139, 720-9.

Jiang XY, Chang FH, Bai TY, Lv XL, Wang MJ (2014). Susceptibility of lung cancer with polymorphisms of CYP1A1, GSTM1, GSTM3, GSTT1 and GSTP1 genotypes in the population of inner Mongolia region. Asian Pac J Cancer Prev, 15, 5207-14.

Kiyohara C, Horiuchi T, Takayama K, Nakanishi Y (2012). Genetic polymorphisms involved in carcinogen metabolism and DNA repair and lung cancer risk in a Japanese population. $J$ Thorac Oncol, 7, 954-62.

Lee MS, Su L, Christiani DC (2010). Synergistic effects of NAT2 slow and GSTM1 null genotypes on carcinogen DNA damage in the lung .Cancer Epidemiol Biomarkers Prev, 19, 1492-7.

Lewis SJ, Cherry NM, Niven MR, Barber PV, Povey AC (2002). GSTM1, GSTT1 and GSTP1 polymorphisms and lung cancer risk. Cancer Lett, 180, 165-71.

Malik MA, Upadhyay R, Mittal RD, et al (2010). Association of xenobiotic metabolizing enzymes genetic polymorphisms with esophageal cancer in Kashmir Valley and influence of environmental factors. Nutr Cancer, 62, 734-42.

Mota P, Silva HC, Soares MJ, et al (2015). Genetic polymorphisms of phase I and phase II metabolic enzymes as modulators of lung cancer susceptibility. J Cancer Res Clin Oncol, 141, 
851-60.

Nasir Uddin MM, Ahmed MU, Islam MS, et al (2014). Genetic polymorphisms of GSTM1, GSTP1 and GSTT1 genes and lung cancer susceptibility in the Bangladeshi population. Asian Pac J Trop Biomed, 4, 982-9.

Naveen AT, Adithan C, Padmaja N, et al (2004). Glutathione S-transferase M1 and T1 null genotype distribution in South Indians. Eur J Clin Pharmacol, 60, 403-6.

Peddireddy V, Badabagni SV, Gundimeda SD, et al (2016). Association of CYP1A1, GSTM1 and GSTT1 gene polymorphisms with risk of non small cell lung cancer in Andhra Pradesh region of South India. Eur JMed Res, 21, 17.

Sharma N, Singh A, Singh N, Behera D, Sharma S (2015). Genetic polymorphisms in GSTM1, GSTT1 and GSTP1 genes and risk of lung cancer in a North Indian population. Cancer Epidemiol, 39, 947-55.

Shukla RK, Tilak AR, Kumar C, et al (2013). Associations of CYP1A1, GSTM1 and GSTT1 polymorphisms with lung cancer susceptibility in a Northern Indian Population. Asian Pac J Cancer Prev, 14, 3345-9.

Vettriselvi V, Vijayalakshmi K, Solomon FDP, Venkatachalam P (2006). Genetic variation of GSTM1, GSTT1 and GSTP1 genes in a South Indian population. Asian Pac J Cancer Prev, 7, 325-8.

\section{(요}

This work is licensed under a Creative Commons AttributionNon Commercial 4.0 International License. 